\title{
Entrenamiento físico y educación como parte de la rehabilitación pulmonar en pacientes con EPOC
}

\author{
KATHERINE MARÍN D.*, REBECA LAUDE P.** y CECILIA MORALES D.***
}

Exercise training and education as part of pulmonary rehabilitation for COPD patients

Pulmonary rehabilitation is a non-pharmacological therapeutic resort for COPD patients. Its main goals are to reduce symptoms, improve quality of life, and increase physical and emotional involvement in everyday activities. COPD patients at all stages of the disease benefit from exercise training programs, as they improve both exercise tolerance and symptoms of dyspnea and fatigue. Patient's education is included so that it helps improving skills, ability to cope with illness, and health status, as well as smoking cessation. We present a bibliographical review about exercise training and education as part of a pulmonary rehabilitation program.

Key words: Pulmonary rehabilitation, COPD, exercise training and education.

\section{Resumen}

La rehabilitación pulmonar es un recurso terapéutico no farmacológico dirigido a pacientes con EPOC, sus principales objetivos son: reducir los síntomas, mejorar la calidad de vida y aumentar la participación física y emocional de los pacientes en las actividades cotidianas. Los pacientes con EPOC en todos los estadios de la enfermedad se benefician con los programas de entrenamiento, que mejoran tanto la tolerancia al ejercicio como la sensación de disnea y fatiga. La educación al paciente se incluye con el objetivo de optimizar sus habilidades, su capacidad de sobrellevar la enfermedad y para mejorar su estado de salud, además de la cesación del hábito tabáquico. A continuación se presenta una revisión bibliográfica acerca del entrenamiento físico y la educación como parte de un programa de rehabilitación pulmonar.

Palabras clave: Rehabilitación pulmonar, EPOC, entrenamiento, educación.

\section{Introducción}

El entrenamiento físico es una parte fundamental de los programas de rehabilitación pulmonar, el cual forma parte del tratamiento no farmacológico de la EPOC estable. La Sociedad Torácica Americana (ATS) y la Sociedad Respiratoria Europea (ERS) definen la rehabilitación pulmonar como “... una intervención multidisciplinaria, basada en la evidencia, dirigida a pacientes con enfermedades respiratorias crónicas, quienes padecen de sintomas y generalmente encuentran disminuidas sus actividades diarias" 1 . Es decir, de acuerdo a la definición, la rehabilitación pulmonar debe ser considerada dentro del tratamiento individual de los pacientes con EPOC que presentan disnea $\mathrm{u}$ otros síntomas respiratorios, tolerancia al ejercicio reducida, restricción en las actividades por causa de la enfermedad o una salud deteriorada ${ }^{1-6}$.

Los objetivos de la rehabilitación pulmonar son: reducir los síntomas, mejorar la calidad de vida, aumentar la capacidad funcional y reducir la utilización de recursos ${ }^{1,2,4}$. Estos objetivos deben ser logrados mediante un programa multidisciplinario personalizado compuesto por entrenamiento físico, educación, nutrición, soporte psicológico y terapia ocupacional $1^{1,3,4,6}$. De todos estos, el entrenamiento físico es el componente más importante ${ }^{1,2,4,6} \mathrm{y}$, junto a la educa-

\footnotetext{
* Facultad de Medicina, Universidad del Desarrollo.

** Consultorio Cordillera, Valparaíso.

*** Consultorio Padre Damián de Molokai, Valparaíso.
} 
ción, son acciones que competen directamente al kinesiólogo.

\section{Disnea y limitación del ejercicio en la EPOC}

La intolerancia al ejercicio y la disnea, definida como “...una experiencia subjetiva de incomodidad respiratoria que se compone de sensaciones cualitativamente diferentes que varían en intensidad", son los síntomas más comunes de la EPOC y aumentan progresivamente a medida que la enfermedad avanza ${ }^{8,9}$. La intolerancia al ejercicio es multifactorial, siendo la limitación ventilatoria el evento predominante en la enfermedad avanzada ${ }^{8}$. Son múltiples los factores que determinan esta limitación ventilatoria, dentro de ellos están las anormalidades integradas entre la mecánica ventilatoria y la función de los músculos respiratorios, así como el aumento en la demanda ventilatoria (como resultado de anormalidades en el intercambio gaseoso) además de alteraciones del control neural de la respiración ${ }^{9}$.

A pesar de la heterogeneidad que caracteriza a la EPOC, existe un denominador común dentro de ella: la limitación al flujo espiratorio. En etapas avanzadas de la enfermedad el aumento de la ventilación durante el ejercicio se produce a expensas de la frecuencia respiratoria $y$, en menor grado, por aumento del volumen corriente. En esta situación, con un tiempo espiratorio menor y con una limitación al flujo espiratorio, se produce atrapamiento aéreo y, consecuentemente, un aumento de la capacidad residual funcional, fenómeno conocido como hiperinflación pulmonar dinámica (HD) $)^{10,11}$. Es así como se ha observado una fuerte relación entre la intensidad de la disnea durante el ejercicio e índices de $\mathrm{HD}^{8}$. La HD altera la relación longitud-tensión de los músculos inspiratorios, especialmente del diafragma, comprometiendo su habilidad para generar presión ${ }^{11,12}$ y la presión intratorácica positiva, además de actuar como una carga inspiratoria umbral, contribuye al deterioro cardíaco observado durante el ejercicio. Así también, la HD produce una disociación neuromecánica, que contribuye a la intensidad de la disnea, al producirse un desequilibrio entre la tensión generada en la activación muscular y los cambios obtenidos en el volumen pulmonar ${ }^{9,10}$.

En pacientes con EPOC severa comúnmente se produce hipoxemia durante el ejercicio debido a la alteración de las relaciones ventilación/ perfusión $(\mathrm{V} / \mathrm{Q})$, a la disminución en la tensión de oxígeno en la sangre venosa, y al efecto de ésta última sobre unidades alveolares con rela- ciones V/Q reducidas. Asimismo, debido al patrón respiratorio rápido y superficial de los pacientes con EPOC avanzada, el espacio muerto fisiológico se encuentra aumentado en reposo y puede incrementarse significativamente durante el ejercicio. Esto último se debe a la restricción mecánica que impone la HD para aumentar el volumen corriente y puede comprometer la eliminación de $\mathrm{CO}_{2}$, determinando hipercapnia inducida por el ejercicio ${ }^{8}$. El deterioro de los gases en sangre estimula en forma excesiva la ventilación, agravando la HD y causando una temprana limitación del ejercicio. Además de la mayor demanda ventilatoria determinada por la hipoxia, la capacidad para realizar ejercicio es afectada también por una menor entrega de oxígeno a los músculos periféricos. El aumento consecuente de la actividad glicolítica anaeróbica incrementa la lactacidemia ${ }^{8}$, favorecida además por la atrofia muscular resultante del círculo disnea-inactividad-desentrenamiento-disnea que presentan estos pacientes ${ }^{13}$. El aumento de la concentración de ácido láctico determina una mayor $\mathrm{PaCO}_{2}$ potenciando la hipercapnia inducida por el ejercicio descrita anteriormente, lo que produce mayor disnea.

\section{EPOC y entrenamiento físico}

Se ha comprobado en numerosos estudios que la rehabilitación pulmonar, y principalmente el entrenamiento físico, producen una mejoría significativa de la disnea, de la capacidad para realizar ejercicio y de la calidad de vida. A pesar de estos beneficios, durante mucho tiempo se ha señalado que la rehabilitación pulmonar, $\mathrm{y}$ específicamente el entrenamiento físico, no provoca cambios en la función pulmonar propiamente tal, ya que no modifica los resultados obtenidos en las pruebas de función pulmonar realizadas con posterioridad al entrenamiento. Sin embargo, se ha observado que al disminuir la demanda ventilatoria, el entrenamiento es capaz de reducir la hiperinsuflación dinámica durante el ejercicio ${ }^{14}$.

El efecto del entrenamiento físico sobre la capacidad para realizar ejercicio y sobre la disnea en los pacientes con EPOC es dependiente de la dosis ${ }^{15}$. Sin embargo, a pesar de que en la mayoría de los programas de rehabilitación pulmonar está incluido el entrenamiento físico, no existe una estandarización de los parámetros de la carga necesarios para lograr los cambios esperados, como hipertrofia de las fibras musculares tipo II, aumento del número de mitocondrias 
y aumento de enzimas que facilitan la fosforilación oxidativa, lo que no ha sido suficientemente estudiado en pacientes con EPOC. Los cambios estructurales y bioquímicos en el músculo producto del ejercicio ocurrirán siempre y cuando el estímulo del entrenamiento en términos de frecuencia, intensidad y duración sea el adecuado $^{14,15}$. La mayoría de los programas incluyen sesiones de ejercicio de 30 minutos o más, 2 a 5 veces a la semana por 6 a 12 semanas $^{3,4}$. Aunque dos veces a la semana es aceptable desde el punto de vista de las mejoras fisiológicas, la frecuencia óptima sería de 3 veces por semana, durante 8 semanas, ya que se logra un equilibrio entre costo del programa y beneficios alcanzados. En cuanto a la relación entre severidad de la enfermedad y duración del entrenamiento, se ha observado que pacientes con EPOC severo se benefician de la rehabilitación durante al menos 6 meses, mientras que en pacientes con EPOC leve a moderado los beneficios se pueden alcanzar con programas de rehabilitación más cortos, de dos o tres meses ${ }^{5}$. Un punto importante se relaciona a la intensidad del ejercicio necesaria para alcanzar la respuesta esperada al entrenamiento. Para los pacientes con capacidad ventilatoria limitada, los criterios basados en la frecuencia cardíaca o el consumo máximo de $\mathrm{O}_{2}\left(\mathrm{VO}_{2}\right.$ máx $)$ para seleccionar la intensidad del entrenamiento son probablemente inadecuados ${ }^{15}$. Por resultar un método mucho más práctico, se recomienda determinar la carga del entrenamiento en base a un porcentaje de la carga máxima alcanzada por el paciente durante una prueba incremental en cicloergómetro. Esta es una prueba sencilla que se puede realizar las veces que sea necesario durante el programa de rehabilitación tanto para evaluar el avance del paciente como para ajustar las cargas de entrenamiento a medida que mejora su condición física.

\section{Planificación del entrenamiento}

En la literatura se describen diferentes tipos de entrenamientos aplicados a pacientes con EPOC: entrenamiento de fuerza, que consiste en trabajar grupos musculares contra alguna resistencia utilizando elementos como pesas o bandas elásticas, con el objetivo de mejorar principalmente el rendimiento de las fibras musculares tipo II, especialmente afectadas por el desentrenamiento que presentan la mayoría de estos pacientes; entrenamiento de resistencia aeróbica como caminar o pedalear, para mejorar principalmente el sistema cardiovascular; y entrenamientos combinados que incluyen los dos tipos mencionados anteriormente y al que en algunos casos se suma el entrenamiento con válvulas inspiratorias. Diferentes estudios han concluido que los tres tipos de entrenamiento tienen efectos beneficiosos en la disnea y en la calidad de vida. El entrenamiento de resistencia aeróbica ha mostrado ventajas sobre el entrenamiento de fuerza en el tratamiento de la EPOC, y a pesar de que el tipo combinado es bien tolerado por los pacientes, pudiendo ser una alternativa válida de entrenamiento, no muestra ventajas adicionales a las de los programas más clásicos de resistencia aeróbica (por ejemplo, sólo trabajar en cicloergómetro), además de implicar mayor costo relacionado con el tiempo y los recursos empleados ${ }^{16,17}$.

Una propuesta interesante de entrenamiento es la modalidad de intervalo, que consiste en realizar el ejercicio a diferentes intensidades dentro de una misma sesión. Así, por ejemplo, un paciente puede pedalear en cicloergómetro durante 5 minutos al $50 \%$ de su carga máxima, continuar con 5 minutos al $70 \%$ y nuevamente 5 minutos al 50\%, repitiendo este ciclo dos o tres veces. Las intensidades y el tiempo que el paciente se mantiene en cada carga van a depender de los resultados de la evaluación que se realiza al inicio del programa. Este tipo de entrenamiento permite alcanzar niveles altos de intensidad, pero imposibles de mantener para la mayoría de los pacientes durante un tiempo prolongado, por lo cual se alternan con intensidades menos exigentes dando la posibilidad a los pacientes de recuperación para completar la sesión de entrenamiento y finalizar con una menor disnea asociada ${ }^{1,3,18}$. Debido a su naturaleza intermitente, otro beneficio de este tipo de entrenamiento, es que se asemeja a la exigencia impuesta por actividades de la vida diaria en pacientes con $\mathrm{EPOC}^{19}$.

En cuanto a la etapa de la enfermedad en la cual se debería comenzar el entrenamiento, se ha observado que en todos los estadios de la enfermedad los pacientes con EPOC se benefician con los programas de rehabilitación ${ }^{1,2,20}$. Además se han comunicado beneficios con programas de rehabilitación dirigidos a pacientes hospitalizados, ambulatorios y en el ámbito domiciliario ${ }^{5}$.

\section{EPOC y educación en salud}

La educación es una parte importante de un programa de rehabilitación, y cada sesión de entrenamiento es una gran oportunidad para, a 
través de una amena conversación, compartir información de utilidad para el manejo de la enfermedad. La educación en salud es un término muy amplio que engloba la totalidad de los aspectos que modifican la enfermedad, incluso antes de su aparición. En el caso de la educación sanitaria dirigida a los pacientes con EPOC, la participación activa en el tratamiento y control de su enfermedad es actualmente el principal objetivo ${ }^{1}$.

La educación a pacientes con EPOC aparece como uno de los componentes principales de los programas de rehabilitación pulmonar ${ }^{1-3,5}$, mencionando como contenidos importantes: información sobre la enfermedad, técnica de inhalación, consejo antitabaco, dieta y ejercicio ${ }^{1,17}$. Aún así, los estudios sobre los efectos de la educación en estos pacientes son escasos.

La educación entregada fuera de un programa integral de rehabilitación pulmonar ha mostrado no ser efectiva, al igual que la educación dirigida sólo a entregar información sobre la enfermedad, la cual no genera cambios en la condición de salud del paciente ${ }^{21}$. La educación para el autocuidado, definida como una forma de educación que apunta a enseñar destrezas para un control óptimo de la enfermedad, alcanzar cambios conductuales y mejorar la adaptación a ésta ${ }^{4}$, es una herramienta que no sólo refuerza las acciones promocionales y preventivas orientadas a mejorar la calidad de vida, modificando sus condicionantes o determinantes, sino que también actúa en los distintos niveles de la enfermedad, tanto en la reducción de los factores de riesgo, como también en las medidas de prevención, tratamiento y rehabilitación del daño en salud. La educación para el autocuidado en pacientes con EPOC ha mostrado tener buenos resultados al mejorar la adherencia al programa de rehabilitación, optimizando la utilización de los fármacos, disminuyendo el número de exacerbaciones y los costos de hospitalización $^{1,22,23}$.

\section{Evaluación de los efectos del programa de rehabilitación}

La evaluación de los resultados ha llegado a ser un componente importante de la rehabilitación pulmonar para determinar la respuesta individual de cada paciente y para determinar la eficacia general del programa. Esta evaluación debe hacerse a través de la comparación de los resultados de cada una de las mediciones realizadas al paciente antes de iniciar y al finalizar el programa de rehabilitación (PR). No existe un modelo de evaluación en los PR que establezca parámetros que deban ser incluidos obligatoriamente durante el programa. Lo extenso de la evaluación, dependerá del propósito de las mediciones, las metas del programa, de los recursos y el nivel de experiencia clínica ${ }^{4,24}$.

Los parámetros evaluados en un PR se pueden categorizar informalmente de acuerdo a la Clasificación Internacional de Deterioro, Discapacidad y Handicap o desventaja desarrollado por la Organización Mundial de la Salud. Bajo esta clasificación, para la EPOC, podemos determinar lo siguiente:

a) Deterioro es la pérdida o anormalidad en la estructura o de la función psicológica, fisiológica o anatómica producto de la enfermedad respiratoria. El deterioro es la exteriorización de un estado patológico, y está determinado generalmente por mediciones de laboratorio. Así, para evaluar el deterioro se puede realizar una espirometría antes de comenzar y después de finalizar el programa, así como medir $\mathrm{VO}_{2}$ máx y el lactato sanguíneo.

b) Discapacidad se refiere a la imposibilidad para realizar una actividad dentro del rango normal esperado a causa de la enfermedad pulmonar. Esto incluiría las limitaciones determinadas por el deterioro en el desempeño físico, como la disnea de esfuerzo. En la rehabilitación pulmonar, la discapacidad es, a menudo, determinada por pruebas tales como la prueba de caminata en 6 minutos, que puede determinar la habilidad del paciente para realizar una actividad específica de la vida diaria como es caminar. Otra importante forma de evaluar la discapacidad en pacientes con EPOC es mediante una prueba de carga submáxima en cicloergómetro que indica la capacidad de resistencia aeróbica al ejercicio.

c) Handicap (desventaja o impedimento) representa la desventaja que resulta de un deterioro o la discapacidad dentro del contexto de la habilidad del paciente para realizarse en la sociedad o cumplir con su rol esperado. Por ejemplo, el desempeño reducido de una persona durante una prueba de marcha en 6 minutos representa su discapacidad, y la resultante incapacidad para mantener su empleo es el handicap. El handicap, o impacto social de la enfermedad, se puede determinar usando mediciones de la calidad de vida relacionada con el estado de salud, para esto se pueden utilizar cuestionarios como por ejemplo el CRDQ o Saint George ${ }^{1,6}$. El concepto de calidad de vida es muy usado porque acerca a la rehabilitación a aquellas áreas de la vida que el paciente puede reconocer como deterioradas. 


\section{Conclusión}

A partir de la evidencia presentada, que demuestra los beneficios tanto físicos, psicológicos como sociales de los programas de rehabilitación pulmonar, sería interesante implementar programas basados en la fisiología del ejercicio, la teoría del entrenamiento y la educación para pacientes con EPOC.

\section{Agradecimientos}

Las autoras agradecen a la Dra. Mónica Gutiérrez C., por su apoyo y asesoramiento en la realización de la presente revisión.

\section{Bibliografía}

1.- NICI L, DONNER C, WOUTER E, ZUMALLACK R, AMBROSTINA N, BOURBEAU J, et al. American Thoracic Society/European Respiratory Society Statement on Pulmonary Rehabilitation. Am J Respir Crit Care Med 2006; 173: 1390-413.

2.- PAUWELS R, BUIST A, CALVERLEY P, JENKINS C R, HURDS S, et al. Global Strategy for the Diagnosis, Management and Prevention of Chronic Obstructive Pulmonary Disease. NHLBI/WHO Global Initiative for Chronic Obstructive Lung Disease (GOLD) Workshop summary. Am J Respir Crit Care Med 2001; 163: 1256-76.

3.- Standards for the diagnosis and treatment of patients with COPD: a summary of the ATS/ERS position paper. Eur Respir J Eur Respir J 2004; 23: 932-46.

4.- TROOSTERS T, CASABURI R, GOSSELINK R, DECMAMER M, et al. Pulmonary Rehabiliatation in Chronic Obstructive Pulmonary Disease. Am J Respir Crit Care Med 2005; 172: 19-38.

5.- SALMAN G, MOSIER M, BEASLEY B, CALKINS, et al. Rehabilitation for Patients with Chronic Obstructive Pulmonary Disease. Meta-analysis of Randomized Controlled Trials. J Gen Intern Med 2003; 18: 213 21.

6.- BRITISH THORACIC SOCIETY. Standards of Care Subcommittee on Pulmonary Rehabilitation. Pulmonary Rehabilitation. Thorax 2001; 56: 827-34.

7.- AMERICAN THORACIC SOCIETY. Dyspnea Mechanisms, Assessment, and Management: A Consensus Statement. Am J Respir Crit Care Med 1999; 159: 321-40.

8.- O'DONNELL E. Ventilatory limitations in chronic obstructive pulmonary disease. Med Sci Sports Exerc 2001; 33 (7): 647-55.

9.- O'DONNELL D, BANZETT R, CARRIERIKOHLMAN V, CASABURI R, DAVENPORT P W.

Correspondencia a:

Dra. Katherine Marín D.

Facultad de Medicina, Universidad del Desarrollo

E-mail:kmarin@udd.cl
Pathophysiology of Dyspnea in Chronic Obstructive Pulmonary Disease. Proc Am Thorac Soc 2007; 4: 145-68.

10.- SUE D. Peripheral Muscle Dysfunction in Patients with COPD. Chest 2003; 124: 1-4.

11.- O'DONNELL D E, SCIURBA F, CELLI B, MAHLER D A, WEBB K A, KALBERG C J, et al. Effect of fluticasone propionate/salmeterol on lung hyperinflation and exercise endurance in COPD. Chest 2006; 130: 647-56.

12.- LISBOA C, BORZONE G, DÍAZ O. Hiperinflación pulmonar en la enfermedad pulmonar obstructiva crónica: Importancia funcional y clínica. Rev Chil Enferm Respir 2004; 20: 9-20.

13.- AMERICAN THORACIC SOCIETY, EUROPEAN RESPIRATORY SOCIETY. Skeletal muscle dysfunction in chronic obstructive pulmonary disease. Am J Respir Crit Care Med 1999; 159: S1-40.

14.- PORSZASZ J, EMTNER M, GOTO S, SOMFAY A, WHIPP B J, CASABURI R. Exercise training decreases ventilatory requirements and exercise-induced hyperinflation at submaximal intensities in patients with COPD. Chest 2005; 128: 2025-34.

15.- CASABURI R, PATESSIO A, IOLI F, ZANABONI S, DONNER C F, WASSERMAN K. Reductions in exercise lactic acidosis and ventilation as a result of exercise training in patients with obstructive lung disease. Am Rev Respir Dis 1991; 143: 9-18.

16.- MONTEMAYOR T, ORTEGA F. Estrategias de Entrenamiento Muscular en la Enfermedad Pulmonar Obstructiva Crónica. ¿Entrenamiento de Resistencia, de Fuerza o Combinado? Arch Bronconeumol 2001; 37: 279-85.

17.- ORTEGA F, TORAL J, CEJUDO P, VILLAGÓMEZ $\mathrm{R}$, SÁNCHEZ H, CASTILLO J, et al. Comparison of effects of strength and endurance training in patients with chronic obstructive pulmonary disease. Am J Respir Crit Care Med 2002; 166: 669-74.

18.- VOGIATZIS I, NANAS S, ROUSSOS C. Interval training as an alternative modality to continuous exercise in patients with COPD. Eur Respir J 2002; 20: $12-9$.

19.- COPPOOLSE A, SCHOLS E, BAARENDS R, MOSTERT R, AKKERMAN M A, JANSSEN P P, et al. Interval versus continuous training in patients with severe COPD: a Randomized clinical trial. Eur Respir J 1999; 14: 258-63.

20.- BERRY M, REJESKI J, ADAIR N, ZACCARO D. Exercise rehabilitation and chronic obstructive pulmonary disease stage. Am J Respir Crit Care Med 1999; 160: 1248-53.

21.- ÁLVAREZ-SALA J, CIMAS E, MASA J, MIRAVITLLES M, MOLINA J, NABERAN K, et al. Recomendaciones para la Atención al Paciente con Enfermedad Pulmonar Obstructiva Crónica. Arch Bronconeumol 2001; 37: 269-78.

22.- BOURBEAU J, JULIEN M, MALTAIS F, ROULEAU M, BEAUPRÉ A, BÉGIN R, et al. Reduction of hospital utilization in patients with chronic obstructive pulmonary disease. Arch Intern Med 2003; 163: 58591.

23.- RAND C S. Patient Adherence with COPD Therapy. Eur Respir Rev 2005 ; 14: 97-101.

24.- DONNER C F, MUIR J F. Selection Criteria and Programmes for Pulmonary Rehabilitation in COPD Patients. Rehabilitation and Chronic Care Scientific Group of the European Respiratory Society. Eur Respir J 1997; 10: 744-57. 